QUALITY IMPROVEMENT REPORT

\title{
Improving assessment of postoperative pain in surgical wards by education and training
}

\author{
R Karlsten, K Ström, L Gunningberg
}

Qual Saf Health Care 2005;14:332-335. doi: 10.1136/qshc.2004.010330

See end of article for authors' affiliations

Correspondence to: Dr R Karlsten, Multidisciplinary Pain Treatment Centre, Uppsala University Hospital, S-751 85 Uppsala, Sweden; rolf. karlsten@astrazeneca.com

Accepted for publication 27 April 2005
Problem: There is a need to improve postoperative pain organisation and management in hospitals. One of the most important factors in achieving this is to improve active assessment of pain in the postoperative phase.

Design: Repeated audits on an annual basis over a 3 year period. Ward nurses, appointed as "pain control representatives", performed the data collection.

Setting: Departments of general surgery and orthopaedics in a university hospital with 1200 beds.

Key measure for improvement: Assessment of postoperative pain intensity using a numerical rating scale. Strategies for change: On the basis of the first audit in 1999 the team decided to introduce a mandatory training programme in postoperative pain management for all involved staff, including surgeons and ward nurses. Guidelines for postoperative care were upgraded and made accessible through the intranet. Regular staff meetings in the surgical wards with representatives from the acute pain service team were introduced.

Effects of change: The assessment of pain according to protocols increased from $71 \%$ to $91 \%$ in the surgical wards and from $60 \%$ to $88 \%$ in the orthopaedic wards between 1999 and $2001 / 2$.

Lessons learnt: To increase the awareness of pain and improve pain assessment, the importance of mandatory training, regular staff meetings and regular audits must be emphasised. It is also imperative to give feedback on the regular audits to the ward and staff members involved.
$\mathrm{P}$ ostoperative pain is a major medical and nursing challenge for many hospitals, even with the development of advanced methods for treating postoperative pain and with increasing knowledge of acute nociceptive pain. ${ }^{1-3}$ The use of advanced techniques for postoperative pain relief increases the need for monitoring, and all staff involved in the care of the patient in the postoperative period should be trained and educated. Important goals for postoperative pain management are to minimise or eliminate discomfort, facilitate the recovery process, and avoid complications. ${ }^{4}$ The role of nurses in surgical care includes assessing the patient's signs and symptoms of pain, carrying out pain relief, and assessing the effects of interventions in the care of the patient. According to the regulations of the Swedish National Board of Health and Welfare, health care should include quality systems for planning, performance, evaluation, and improvement of the given care, and all staff should be involved in this work. ${ }^{5}$

A quality system is defined in terms of organisational structure, routines, processes, and resources that are necessary for managing good quality in health care. The regulation also emphasises the dignity, integrity, participation, and safety of patients. For important processes, there should be measurable goals, indicators, and audit routines. Furthermore, Swedish nurses as well as physicians are required by law to document care-including assessments of care needs, planned and implemented care interventions, and outcomes of care. ${ }^{67}$ National guidelines for postoperative pain management emphasise the importance of distinct pain documentation in the records using the specific keyword "pain". 8

\section{DESCRIPTION OF CONTEXT}

One way to meet these demands is the introduction of an anaesthesiology based acute pain service. ${ }^{9}$ This service has been introduced in many countries and an increasing number of hospitals now provide the service. ${ }^{1}$ The use of an acute pain service based on staff anaesthesiologists, together with specially trained nurses and physiotherapists who provide a high technology pain management service, often has a considerable impact on pain management on the surgical wards. The disadvantage is that only a small percentage of the patients with postoperative pain receive these benefits, so there is a need for increased coverage of this service..$^{11}$

To expand the coverage of the acute pain service, Rawal ${ }^{10}$ has developed a model in Sweden which is described as a nurse based, anaesthesiologist supervised pain service in which acute pain nurses provide training and assistance for ward nurses. The role of the ward nurses is upgraded and highlighted to improve assessment of pain intensity, to administer analgesics, and to monitor efficacy and adverse events. In each surgical ward, nurses and surgeons with a special interest in pain management are appointed "pain control representatives" and have the responsibility for pain management. An important role for the nurse based, anaesthesiologist supervised acute pain service team is to provide guidelines for pain management in cooperation with the surgeons (box 1). ${ }^{31012}$

The University Hospital in Uppsala has 1200 beds and cares for 55000 inpatients each year. In 2002, 7350 surgical procedures requiring assistance by an anaesthesiologist were performed in the departments of general surgery and orthopaedics. These two departments have nine wards, each with 25-30 beds and staffed with 15-20 registered nurses.

At the University Hospital in Uppsala a nurse based, anaesthesiologist supervised pain service started in 1991. Clinical guidelines for pain management were developed and implemented, followed by the introduction of "pain control representatives". The intention was to have a minimum of two registered nurses and a physician from the regular staff as pain control representatives in all hospital wards. The selection of pain control representatives was based on 
Box 1 Pain control representatives at the University Hospital, Uppsala

- A surgical ward with $20-25$ beds is staffed with 15-20 registered nurses.

- Each ward should have a minimum of two registered nurses and one surgeon from the regular staff appointed as pain control representatives.

- The pain control representatives are responsible for dissemination of information to colleagues, as well as education and training in pain management.

- They also participate in collecting data during audits.

personal interest and staff volunteering for the task. Even if some staff changed positions during the observation period, others replaced them so that the minimum standard of one physician and two nurses as pain representatives in each ward could be continued.

Emphasis has been on making pain visible by pointing out the need for assessment and documentation of pain intensity. In 1998, quality goals and indicators for pain management were established at a hospital level. This included acute and postoperative pain, long lasting pain associated with cancer and chronic pain, as well as pain during different kinds of procedures such as radiography. Specific goals for postoperative pain management included regular pain assessment using a numerical rating scale with two end points $(0=$ no pain and $10=$ worst possible pain $)$. Pain with a score of more than 3 on the numerical rating scale should be evaluated with the patient, treated pharmacologically or with alternative methods, and evaluated and documented regularly in the patient record. All patients with postoperative pain should be assessed at least every 4 hours using the numerical rating scale. When the situation is under control, pain assessment should be documented at least three times daily until pain treatment is terminated.

The continuous development of postoperative pain treatment in a hospital must be considered as a chain of events where introduction of pain management methods, training, and education should be evaluated by regular follow up by, for example, audits. The only way to visualise postoperative pain and to be able to treat it promptly is by actually asking patients if they have pain. It is essential that regular assessments of postoperative pain are performed in the postoperative period.

This paper reports the development of a mandatory training programme, including theoretical and practical examinations, and its impact on pain assessment and documentation. The evaluation is based on three consecutive audits performed in the departments of general surgery and orthopaedic surgery.

\section{OUTLINE OF PROBLEM}

In 1999 we conducted a follow up of the described quality goals and indicators for postoperative pain management. An audit of patient records was designed with two pain control representative (nurses) from each ward collecting the data. The reason for this approach was threefold: (1) to empower the pain control representatives to highlight the wards' ownership of the results; (2) to create an environment for change; and (3) to introduce the concept of quality improvement in pain treatment.

The records of 30 patients from each of the nine wards $(n=270)$ were selected consecutively. Inclusion criteria were general surgery or orthopaedic surgery of any kind, both emergency and elective surgery. A data collection form was developed based on recommendations from EuroPain, ${ }^{13}$ including the following quality indicators:

- preoperative information about pain assessment with the numerical rating scale;

- regular pain assessment and recording of pain intensity in the postoperative period;

- treatment and evaluation of treatments in patients with pain intensity $>3$ on the numerical rating scale.

Data regarding age, sex, type of surgery, and pain treatment method were also registered. Written information regarding the audit was given to all head nurses on the wards and to the heads of the departments.

The audit in 1999 showed that only $68.5 \%$ (185/270) of the postoperative patients were assessed using a numerical rating scale according to protocols (every 4 hours during the first 24 hours). The proportion on the surgical wards was $71 \%$ $(149 / 210)$ and $60 \%(36 / 60)$ on the orthopaedic wards. $75 \%$ of the numerical rating scale assessments of $>3$ were treated pharmacologically and $45 \%$ were evaluated with a repeated assessment using the numerical rating scale.

\section{STRATEGIES FOR CHANGE}

\section{Education and training programme for all staff}

To improve postoperative pain management several actions were undertaken. The result from the audit was presented and discussed with the pain control representatives on the wards (both nurses and surgeons). An education and training programme for all nurses and surgeons was developed and implemented after the audit. Educational material to read was given when the course started. This consisted of all protocols and standard orders for pain management including oral patient information, pain assessment, and standard pain treatments (intravenous opioids, patient controlled analgesia, and epidural analgesia).

The course content included basic theoretical training and practical training with infusion pumps as well as discussions based on case related problems. It was divided into three sessions each of 4 hours. At the end of the course a written theoretical and practical test was performed. The written theoretical test included questions on basic pharmacology and on the protocols and standing orders and was undertaken by surgeons and nurses. The practical test concentrated on the use of pumps and was mandatory for nurses since they are responsible for preparing and setting up the treatment after instructions from the prescribing physician. The initial failure rate was $5 \%$ and all who failed the test were re-examined. At completion of the tests a certificate was issued which was valid for 3 years, after which recertification is required. Since 2000 this course has been conducted 2-4 times a year with 20-30 registered nurses from surgical and orthopaedic wards in each course. The course has been mandatory for nurses and newly employed surgeons. Surgeons acting as pain control representatives have had an important role in spreading the information to other surgeons at internal meetings.

\section{Network and seminars for pain control representatives}

The pain control representatives were invited to meetings at least every second month with the acute pain service team to exchange information and experiences. Furthermore, 1 day seminars were conducted twice a year for both pain control representatives and all other staff interested in pain management. These 1 day seminars were used to refresh the basic knowledge of pain physiology and treatment. Themes such as postoperative management, cancer related pain, and 
management of addiction were other items discussed at these meetings. The information given at these meetings was communicated to the wards by the pain control representatives. The guidelines for postoperative pain management were updated annually and made accessible through the hospital intranet.

\section{Patient information and education}

In the wards, emphasis was put on improving information to patients about postoperative pain, different methods for treatment, and the importance of active participation by patients in their treatment. A brochure was developed by the pain control representatives and introduced in late 2000, providing written information on pain and pain assessment and stressing the importance of the patient's participation in the treatment. The brochure was used as a complement to verbal information provided by ward nurses and was sent to patients to read before admission to hospital.

\section{EFFECTS OF CHANGE}

In the following years, new audits have been performed using the described methodology. As a result of structural reorganisation at the hospital, one ward was excluded compared with the audit in 1999. In $2000(n=239)$, pain assessment was performed according to protocol essentially as in the preceding year in $72 \%$ of cases (173/239). However, in the latest audit 2001/2 $(n=235)$ the use of pain assessment had increased and was performed according to protocol in $90 \%$ of cases (fig 1). On the general surgery wards 91\% (159/175) were assessed according to protocols compared with $88 \%$ (53/60) of the cases on the orthopaedic wards. The increase in assessment of pain is statistically significant when the audits of 2000 and 2001/2 are compared $\left(\chi^{2}\right.$ test $\left.24.69(\mathrm{df}=1), \mathrm{p}<0.01\right)$.

The selection of the specific method of postoperative pain treatment is mainly based on a joint clinical evaluation by the responsible surgeon and anaesthesiologist. The standard methods for postoperative pain relief used were as follows: oral analgesics $19 \%$ (44/235), intermittent opioid injection $37 \%$ (87/235), patient controlled analgesia 16\% (38/235), and epidural analgesia $28 \%(66 / 235)$. In the groups treated with patient controlled analgesia or epidural analgesia, 43\% (45/ 104) of the patients underwent surgery for cancer and $24 \%$ $(25 / 104)$ had major orthopaedic or vascular surgery. Analysis of the assessment of pain according to method of treatment showed that patients with epidural analgesia and patient controlled analgesia were most often assessed according to protocol. No pharmacological treatment was administered to $33 \%$ of patients reporting pain with a value of $>3$ on the numerical rating scale. In some cases other actions were taken, such as changing the position in bed. However, a detailed analysis was not possible on the basis of the available information.

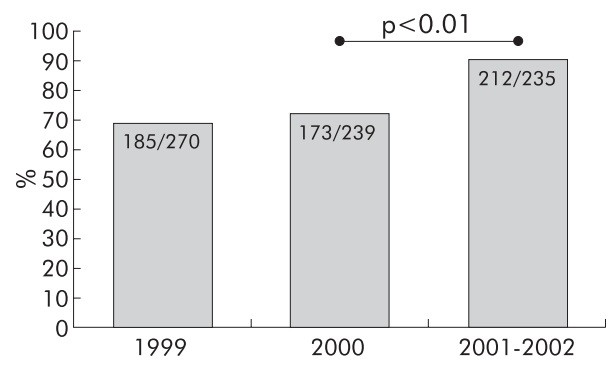

Figure 1 Percentage of patients assessed for postoperative pain according to protocol in three consecutive audits.

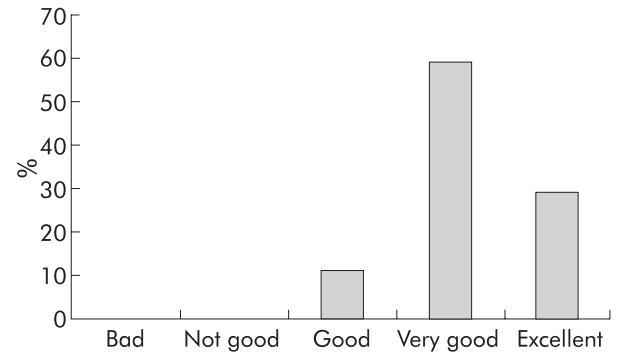

Figure 2 Overall rating of postoperative pain treatment in patients undergoing major surgery for cancer: 38 of 45 patients responded.

Details from the last audit (2001/2) reveal additional data. According to the national guidelines ${ }^{8}$ and guidelines issued by EuroPain ${ }^{13}$ for postoperative pain management, pain at rest and movement was added to the audit as well as a question to the patient regarding their global rating of the postoperative pain relief using a 5-step Likert scale. Most patients $(97 \%)$ rated postoperative pain treatment as good, very good or excellent, and only 3\% rated the postoperative pain treatment as not good or bad. A special analysis was made in patients who underwent major surgery for cancer $(\mathrm{n}=45)$. The most common procedures were prostatectomy, colectomy, cystectomy, and nephrectomy. All of these patients had patient controlled analgesia $(n=20)$ and/or epidural analgesia $(n=36)$. The reason for adding patient controlled analgesia to an epidural analgesia $(\mathrm{n}=11)$ was preoperative treatment with high doses of opioids. All of these patients had their pain assessed according to protocols and their overall rating of the postoperative pain control was high (fig 2).

\section{LESSONS LEARNT}

The audits have clearly shown the importance of regular follow up, training, and education in improving pain assessment and the care of patients with postoperative pain. Regular audits also highlight the importance of pain treatment for all staff members involved. The implementation of pain control representatives on all wards is a means of strengthening the culture and awareness for pain treatment in everyday work. Regular documentation of the patients' pain score enforces some kind of action. In some cases this might be changing position in the bed while in others it includes some medical intervention.

The education and training programme for all staff is a heavy burden for all involved. Repetitive training is challenging for those who act as teachers, and the workload on all staff members with limited time for training and education is another issue. Involving physicians is a special challenge; all newly employed physicians are trained in the programme but, for practical reasons, senior physicians were informed and educated by local meetings at the wards arranged by the acute pain service as well and through their respective pain control representatives. An important experience gained during this period was that all staff involved in the care and treatment of the patient need to be updated and continuously informed about the guidelines in order for the programme to be successful. The pain control representatives are very important in the process since they have daily and regular contact with the ward and all staff members. We continue to strengthen the role of pain control representatives as facilitators and also their essential role in quality improvement.

Patients' pain has become visible through regular pain assessment and documentation according to the quality goals of the hospital. Our results can be compared with a recent 
Swedish study ${ }^{14}$ which showed that less than $10 \%$ of patient records $(n=172)$ contained notes on systematic assessment with a pain assessment instrument.

In 1998 and 2002 Kitson and colleagues presented a conceptual framework for implementation of research findings in practice. ${ }^{15} 16$ The interplay and interdependency of three major components were highlighted:

- the nature of the evidence being used;

- the quality of context in terms of its ability to cope with change; and

- the type of facilitation needed to ensure a successful change process.

It could be assumed that both physicians and nurses agree that the patient should receive adequate and evidence based pain management. However, during the present quality improvement process, high workload pressure and staff turnover-as well as changing leadership-have had a negative influence on the ability of the departments to cope with the change process. In some wards only around half of the nursing staff had worked more than a year. Consequently, the pain control representatives and the acute pain service team have had a very important role in maintaining standards. The acute pain service has served as an external facilitator providing guidelines, methods, goals, and feedback of audits for learning purposes, not for judgement. They have also provided channels for dialogue and have the mandate to discuss and change necessary parts of the system. The pain control representatives, as internal facilitators, have adopted and disseminated ideas in their wards. This reflects the conclusion of Burrows in a literature review of facilitation ${ }^{17}$ that genuine mutual respect, a partnership in learning, a dynamic goal orientated process, and critical reflection are essential for effective facilitation.

The patients in the audited departments were satisfied with their pain management. Idvall ${ }^{18}$ found that postoperative patients were very satisfied with the pain relief even if they reported severe pain in the previous 24 hours. Explanations were that the patients anticipated having postoperative pain and wanted to be considered cooperative. None of the patients blamed the staff for their severe pain. This has been seen in other studies such as that by Meehan et $a l^{19}$ and, consequently, the level of pain as measured by a visual analogue scale or numerical rating scale does not correlate with the satisfaction of patients with their medical care. Patient satisfaction cannot therefore be used as the sole indicator of an effective pain service in hospitals. ${ }^{13}$ In a 10 year review of quality improvement monitoring in pain management in the US, the following indicators are recommended $^{20}$ :

- pain intensity should be documented with a numerical or descriptive rating scale;

\section{Key messages}

- Pain has to be visualised, and assessing pain in the postoperative period is essential for improving pain management.

- Pain control representatives are important as facilitators and for communicating results from audits, for implementing changes in care, and to increase awareness of pain as a problem in everyday care.

- Regular audits are mandatory for the continuous development of patient care.
- pain intensity should be documented at frequent intervals;

- pain should be treated by a route other than the intramuscular route:

- pain should be treated with regularly administered analgesics;

- pain should be prevented and controlled to a degree that facilitates function and quality of life; and

- patients should be adequately informed and knowledgeable about pain management.

\section{NEXT STEPS}

The quality improvement project described here has initiated an ongoing study with the aim of describing and comparing assessments by patients and nurses of the quality of postoperative pain management including the recommended indicators described above. ${ }^{20}$ We also plan to analyse the reasons for not administering pharmacological treatment in patients indicating moderate to severe postoperative pain.

\section{Authors' affiliations}

R Karlsten, Multidisciplinary Pain Treatment Centre, Uppsala University Hospital, Uppsala, Sweden

K Ström, Department of Anaesthesiology and Intensive Care, Uppsala University Hospital, Uppsala, Sweden

L Gunningberg, Department of Public Health and Caring Sciences,

Section of Caring Sciences, Uppsala University, Uppsala, Sweden

L Gunningberg, Surgery Centre, Uppsala University Hospital, Uppsala,

Sweden

\section{REFERENCES}

1 Werner MU, Soholm L, Rotboll Nielsen P, et al. Does an acute pain service improve postoperative outcome? Anesth Analg 2002;95:1361-72.

2 Carr EC. Refusing analgesics: using continuous improvement to improve pain management on a surgical ward. J Clin Nurs 2002;11:743-52.

3 Rawal N. Acute pain services revisited: good from far, far from good? Reg Anesth Pain Med 2002;27:117-21.

4 International Association for the Study of Pain (IASP). Management of acute pain: a practical guideline. Seattle: IASP Publications, 1992.

5 Socialstyrelsens författningssamling (SOSFS). Kvalitetssystem inom hälso- och sjukvården (Regulations and advisory instructions on quality in health care systems). In Swedish. SOSFS 1996:24. Stockholm: Socialstyrelsen, 1996.

6 Svensk Författningssamling. Patientjournallagen (The Patient Record Act). In Swedish. Stockholm: Liber, Allmänna Förlaget, 1985.

7 Socialstyrelsens Författningssamling (SOSFS). Omvårdnad inom hälso- och sjukvården (Adivisory instruction on nursing). In Swedish. SOSFS 1993:17. Stockholm: Socialstyrelsen, 1993.

8 Medicinska Kvalitetsrådet (MKR). Behandling av postoperativ smärta Riktlinjer och kvalitetsindikatorer (in Swedish). Stockholm: Svensk Medicin 70, Gothia 2001.

9 Ready LB, Oden R, Chadwick HS, et al. Development of an anesthesiologybased postoperative pain management service. Anesthesiology 1988;68:100-6.

10 Rawal N. 10 years of acute pain services: achievements and challenges. Reg Anesth Pain Med 1999;24:68-73.

11 Hung CT, Lau LL, Chan CK, et al. Acute pain services in Hong Kong: facilities, volume, and quality. Hong Kong Med J 2002;8:196-201.

12 Rawal N, Berggren L. Organization of acute pain services: a low-cost model. Pain 1994;57:117-23.

13 European Task Force. European minimum standards for the management of postoperative pain. Goring on Thames: Pegasus Healthcare International Ltd, 1998.

14 Idvall E, Ehrenberg A. Nursing documentation of postoperative pain management. J Clin Nurs 2002;11:734-42.

15 Kitson A, Harvey G, McCormack B. Enabling the implementation of evidence based practice: a conceptual framework. Qual Saf Health Care 1998;7:149-58.

16 Rycroft-Malone J, Kitson A, Harvey G, et al. Ingredients for change: revisiting a conceptual framework. Qual Saf Health Care 2002; 1 1:174-80

17 Burrows D. Facilitation: a concept analysis. J Adv Nurs 1997;25:396-404

18 Idvall E. Post-operative patients in severe pain but satisfied with pain relief. J Clin Nurs 2002;11:841-2.

19 Meehan DA, McRae ME, Rourke DA, et al. Analgesic administration, pain intensity, and patient satisfaction in cardiac surgical patients. Am J Crit Care 1995; 4:435-42.

20 Gordon D, Pellino T, Miaskowski C, et al. A 10-year review of quality improvement monitoring pain management: recommendations for standardized outcome measures. Pain Manage Nurs 2002;3:116-30. 